\title{
Relaxation of excited electrons in an electron gas: A mean-field approach with charge and spin polarizations
}

\author{
I. Nagy, ${ }^{1,2}$ M. Alducin, ${ }^{3}$ and P. M. Echenique ${ }^{4,5}$ \\ ${ }^{1}$ Department of Theoretical Physics, Institute of Physics, Technical University of Budapest, H-1521 Budapest, Hungary \\ ${ }^{2}$ Donostia International Physics Center, 20080 San Sebastián, Spain \\ ${ }^{3}$ Departamento de Ingeniería Eléctrica, E.T.S.I.I., Universidad del País Vasco, Alda. Urquijo s/n, 48013-Bilbao, Spain \\ ${ }^{4}$ Departamento de Física de Materiales, Universidad del País Vasco, Aptdo. 1072, 20080 San Sebastián, Spain \\ ${ }^{5}$ Centro Mixto CSIC-UPV/EHU, Aptdo. 1072, 20080 San Sebastián, Spain
}

(Received 26 October 2001; revised manuscript received 23 January 2002; published 23 May 2002)

\begin{abstract}
The relaxation rate of excited electrons in a paramagnetic electron gas is calculated. The theory is based on the charge and spin response functions and the local-field methodology of generalized mean-field descriptions. A link between this treatment and the kinetic treatment for the relaxation phenomena is established in the first-order Born limit. The relative weight of the spin-fluctuation channel in the important low-energy dynamics is quantified for our model system.
\end{abstract}

DOI: 10.1103/PhysRevB.65.235102

PACS number(s): 71.10.Ca, 72.15.Lh, 71.45.Gm

\section{INTRODUCTION AND MOTIVATIONS}

The dynamical probing of correlated motions of the constituents of a many-body fermion system is currently an active subfield in physics. It is the subject of experiments based on a variety of sophisticated methods and gives the challenge for theoretical attempts. The natural interplay between these basic aspects of understanding needs systematic investigations of the underlying key ingredients on both sides.

The lifetime of single-particle excited states is a fundamental quantity. It can be defined by the imaginary part of the proper self-energy of the one-particle Green's function. ${ }^{1-3}$ This Green's function $(G)$ characterizes the propagation of a disturbance in which a single particle is added to the many-body system. The imaginary part of the self-energy has a direct physical interpretation. It describes the dynamical effect of interparticle interactions, i.e., how the interactions transfer particles from a given (by momentum, energy, and spin) quantum state to another. The excited one-particle states are only approximate eigenstates due to the influence of interparticle interactions on the added particle.

In an electron gas, a physical model of metals, the field particles have their own, charge and spin degrees of freedom. In this model, the use of well-motivated shielded interactions corresponds to accepting the classical principle that an added particle in the interacting system responds to the total field produced by its disturbance in the medium. The consideration via the response function method, i.e., including in principle all kind of relevant excitations, rests on the true physical vacuum of the interacting system.,

It is, therefore, reasonable to expect that the imaginary part of the self-energy expressed in terms of response functions, would give physically sensible results. Physical intuitions, that are also needed in a more formal graphical (diagrammatic) expansion method, are transparently controlled via standard self-consistency (mean-field) arguments in the construction of spectral functions.

This way of construction should be an appropriate one for electrons influenced by the ionic (lattice) external field in real solid targets. Here is, in fact, one of our basic motivations. There have been recent attempts ${ }^{6-8}$ to implement the so-called $G W$ method $^{9}$ using the band paradigm and considering (with $G=G^{0}$ ) the charge-only polarization effect to the imaginary part of the self-energy.

For an excitation phenomena an important question is the true role of band-related ${ }^{10}$ one-particle and different manybody correlation effects. ${ }^{11-14}$ This paper deals with these effects in a paramagnetic electron gas, therefore the obtained results may provide a comparative-background for future band-based calculations using the same level for the response functions.

The paper is organized as follows. In Sec. II, the applied theoretical framework will be outlined and implemented for paramagnetic (metallic) electron systems. This section contains our results in forms of illustrative figures, a table, and analytical expressions. A nontrivial link between the kinetic, scattering approach and the dissipative-medium-based treatment is established there. The last section, Sec. III, is devoted to a short summary and few clarifying comments. Hartree atomic units, $e^{2}=\hbar=m_{e}=1$, are used in this work.

\section{THEORY AND RESULTS}

The simplest approximation that takes into account the long-range screening is the random phase approximation (RPA). The approximation is equivalent ${ }^{15}$ to the timedependent, linearized, and self-consistent, Hartree method for a given Coulomb external field ${ }^{16} W=W_{\mathrm{RPA}}$. In their pioneering ${ }^{17}$ paper on application of field-theoretic methods Quinn and Ferrell used this approximation in order to characterize real transitions and thus the lifetime in a homogeneous electron gas. ${ }^{18,19}$

Beyond the original dielectric (charge-only response) RPA description of the dynamic response function one should consider, even in an unpolarized electron gas, the effects of vertex corrections due to both charge and spin fluctuations. The relevant method, termed now as local-field methodology in self-consistent (generalized mean-field) attempts, is 
established ${ }^{20-24}$ and gives the theoretical framework to the present paper.

The imaginary part of the self-energy of an excited singleparticle is expressed ${ }^{16}$ as an integral over the wave vectors of energetically allowed excitations in the interacting electron gas. In the adopted, denoted as a $G^{0} W$-type, method $W$ is the total effective field due to charge and spin polarizations in the system and $G^{0}$ stands for the free-particle propagator. The induced parts of this field depend quadratically on the coupling strengths, due to the linear-response approximation. ${ }^{20-24}$ The probabilities ${ }^{9}$ for real transitions are described via the imaginary part of the (induced) effective interaction, i.e., the dynamical response functions for real transitions.

Considering these transitions in a paramagnetic electron gas (where there is not mixed charge-spin response, ${ }^{24}$ at least in the adopted linear-response treatment) one can write the relaxation rate $(1 / \tau)$ as the sum of partial rates $[(1 / \tau)$ $\left.=\left(1 / \tau_{c}\right)+\left(1 / \tau_{s}\right)\right]$ governed by the imaginary parts of charge $(c)$ and spin $(s)$ response functions. The partial rates are, therefore, given by

$$
\begin{aligned}
& \frac{1}{\tau_{c}}=\frac{1}{2 \pi^{2}} \frac{1}{v} \int_{0}^{E-E_{F}} d \omega \int_{q_{-}}^{q_{+}} d q q\left|V_{c}(q)\right|^{2} \operatorname{Im} \chi_{c}(q, \omega), \\
& \frac{1}{\tau_{s}}=\frac{3}{2 \pi^{2}} \frac{1}{v} \int_{0}^{E-E_{F}} d \omega \int_{q_{-}}^{q_{+}} d q q\left|V_{s}(q)\right|^{2} \operatorname{Im} \chi_{s}(q, \omega),
\end{aligned}
$$

where the $\chi_{i}(q, \omega)$ functions $(i=c, s)$ are the screened response functions. The frequency-integral is constrained by the Pauli exlusion principle (see, i.e., Ref. 9) and the physical convention $\operatorname{Im} \chi_{i}(q, \omega) \geqslant 0$ for $\omega \geqslant 0$ has been used to write Eqs. (1), (2). $E=v^{2} / 2$ is the energy of an excited electron and the prefactor (3) in Eq. (2) refers to the sum of one longitudinal and two transversal modes. ${ }^{20-24}$ Note, that in writing Eqs. (1) and (2) we have imposed the energy conservation $\left(\omega=\mathbf{q} \cdot \mathbf{v}-q^{2} / 2\right)$ between the external (excited) and intermediate states; we are considering real processes for a measurable fundamental quantity: $q_{ \pm}=\sqrt{2}(\sqrt{E} \pm \sqrt{E-\omega})$.

The coupling to the charge and spin fluctuations are characterized $^{22}$ by the following, instantaneous interactions:

$$
\begin{gathered}
V_{c}(q)=v(q)\left[1-G_{c}(q)\right], \\
V_{s}(q)=-v(q) G_{s}(q),
\end{gathered}
$$

in which $v(q)=4 \pi / q^{2}$, and the negative signs of $v(q) G_{i}(q)$ refer to their basically exchange-mediated origin. The $G_{c}(q)$ and $G_{s}(q)$ functions are local-field factors, related to statistical and dynamical correlations in a well-known manner. ${ }^{20}$ The Fermi energy $E_{F}$ and the Fermi velocity $v_{F}=\sqrt{2 E_{F}}$ are defined via the Wigner-Seitz radius $\left(r_{s}\right)$ in the usual way for the investigated $3 \mathrm{D}$ electron gas of number-density $n_{0}$. Note, that the $V_{c}(q)=v(q)$ and $V_{s}(q)=0$ approximation to Eqs. (1), (2) would correspond to a dielectric (spin-symmetric response to an external scalar field) description, with a dielectric screening [see below, and in point (d)] for the electrontest charge interaction.
Within the generalized mean-field treatment ${ }^{22}$ the screened response functions are represented as

$$
\begin{aligned}
& \chi_{c}(q, \omega)=\frac{\chi_{0}(q, \omega)}{1+V_{c}(q) \chi_{0}(q, \omega)}, \\
& \chi_{s}(q, \omega)=\frac{\chi_{0}(q, \omega)}{1+V_{s}(q) \chi_{0}(q, \omega)} .
\end{aligned}
$$

The $\chi_{0}(q, \omega)$ function is well known, $, 2,16$ and we use its form at $T=0$ temperature. The denominator of Eq. (5) is the electron $(e)$ dielectric function $\epsilon_{e}(q, \omega)=1+V_{c}(q) \chi_{0}(q, \omega)$. Finally, the convenient representations for the local-field factors $\operatorname{are}^{21}$ as follows:

$$
\begin{aligned}
& G_{c}(q)=\frac{1}{2}\left(\frac{q^{2}}{q^{2}+\alpha_{\uparrow \uparrow}^{2}}+\frac{q^{2}}{q^{2}+\alpha_{\uparrow \downarrow}^{2}}\right), \\
& G_{s}(q)=\frac{1}{2}\left(\frac{q^{2}}{q^{2}+\alpha_{\uparrow \uparrow}^{2}}-\frac{q^{2}}{q^{2}+\alpha_{\uparrow \downarrow}^{2}}\right) .
\end{aligned}
$$

In the present study we shall use the theoretical values for the $\alpha_{\uparrow \uparrow}$ and $\alpha_{\uparrow \downarrow}$ factors as given by Iwamoto and Pines. ${ }^{25}$ Technically, the RPA corresponds to Eq. (1), with $G_{c}(q)$ $=0$.

(a) First, we investigate the physically simple but still interesting case of applying a model $(m) V_{m}(q) \equiv 4 \pi C$ constant value in Eq. (1) for the screened interaction (the residual interaction is modeled by a scattering length, as in the Bethe-Salpeter ladder treatment ${ }^{1}$ for an imperfect Fermi gas) together with the ideal response function for the imaginary part. By using the standard [see, Ref. 2, Eq. (5.5.13)] representation for $\operatorname{Im} \chi_{0}(q, \omega)$ at $T=0$, rewriting the resulting Eq. (1) by dimensionless variables, and performing the integrations one obtains the following form:

$$
\frac{1}{\tau_{m}}=\frac{v_{F}^{4}}{\pi} C^{2} \frac{8}{15 \sqrt{x}}\left[\Theta(2-x)(2-x)^{5 / 2}+\frac{5 x-7}{2}\right],
$$

in which $x \equiv E / E_{F}$ and, of course, $x \geqslant 1$. The generalized function $\Theta(u)$ is introduced for short-hand notation in this exact expression. The low-energy asymptotics $\left(x \rightarrow 1^{+}\right)$is proportional to $(x-1)^{2}$, reflecting the robust constraint of the Pauli principle for real transitions at $T=0$. The analytical expression allows useful estimations once the value of $C$ is fixed. It may also allow different, i.e., linearized approximations of the form of $\tau_{m}^{-1}=b_{1}+b_{2}(x-1)$, in different (and finite) ranges of the $x$ variable (see, below at Fig. 2). But, at least in our physical model, such an empirical ${ }^{26}$ approximation may not herald deviations from the normal-state (Landau) behavior. At $x \gg 1$ one has a "gas-kinetical," $4 \pi C^{2} n_{0} v$, character for $\tau_{m}^{-1}$.

(b) Next, for the physically important $\left(E-E_{F}\right) \ll E_{F}$ range, the frequency integrals are analytical, due to $\operatorname{Im} \chi_{i}(q, \omega) \propto \operatorname{Im} \chi_{0}(q, \omega)=\omega /(2 \pi q)$. The remaining $q$ integrations $\left(0 \leqslant q \leqslant 2 v_{F}\right)$ are performed by computer in Eqs. (1) and (2). Table I contains (with the adopted $\alpha_{\uparrow \uparrow}$ and $\alpha_{\uparrow \downarrow}$ values) our detailed results to $\left(1 / \tau_{i}\right)$ for the cases of $i$ 
TABLE I. Theoretical $a_{i}\left(r_{s}\right)$ prefactors $(i=\mathrm{RPA}, c, s)$ to the low-energy expression for $1 / \tau_{i}$ of Eq. (10) as a function of $r_{s}$. The ratio, defined as $R=\left(a_{c}+a_{s}\right) / a_{c}$, shows the enhancements. The $\alpha_{\uparrow \uparrow}$ and $\alpha_{\uparrow \downarrow}$ factors are taken from Ref. 25. See text for further details.

\begin{tabular}{lcccccc}
\hline \hline$r_{s}$ & $a_{\mathrm{RPA}}$ & $\alpha_{\uparrow \uparrow}$ & $\alpha_{\uparrow \downarrow}$ & $a_{c}$ & $a_{s}$ & $R$ \\
\hline 1 & 0.068 & 2.83 & 7.91 & 0.064 & 0.007 & 1.11 \\
2 & 0.384 & 1.44 & 3.17 & 0.345 & 0.101 & 1.29 \\
3 & 1.044 & 0.97 & 1.88 & 0.919 & 0.486 & 1.53 \\
4 & 2.111 & 0.73 & 1.30 & 1.831 & 1.549 & 1.84 \\
5 & 3.626 & 0.59 & 0.99 & 3.121 & 3.755 & 2.20 \\
\hline \hline
\end{tabular}

$=\mathrm{RPA}, c, s$, and $\tau^{-1} \equiv \tau_{c}^{-1}+\tau_{s}^{-1}$. One can use the calculated $a_{i}\left(r_{s}\right)$ factors $\left[a\left(r_{s}\right) \equiv a_{c}\left(r_{s}\right)+a_{s}\left(r_{s}\right)\right]$ in the usual ${ }^{19}$ limiting expression

$$
\frac{1}{\tau_{i}}=a_{i}\left(r_{s}\right)\left(E-E_{F}\right)^{2},
$$

as multiplying factors. The last column of Table I shows the important role of spin fluctuations via $R=a\left(r_{s}\right) / a_{c}\left(r_{s}\right)$. To an acceptable semiquantitative accuracy we can write $a_{\mathrm{RPA}}\left(r_{s}\right) \cong 0.068 r_{s}^{5 / 2}$ and $a\left(r_{s}\right) \cong 0.068 r_{s}^{5 / 2}\left(1+0.035 r_{s}^{2}\right)$, for the investigated metallic range of the density parameter $r_{s}$.

Now, according to the motivation, we compare our detailed results with those obtained ${ }^{27}$ in a band-structure-based $G^{0} W_{\text {RPA }}$ calculation (i.e., using the charge-only dielectric response of real targets) for $\tau_{\mathrm{RPA}}^{b}$. The effect of bands $(b)$ on the homogeneous electron gas equivalent $\tau_{\mathrm{RPA}}$ is about 25 $-35 \%$ reduction in cases of $\mathrm{Al}\left(r_{s}=2\right)$ and $\mathrm{Mg}\left(r_{s}=2.6\right)$ free-electron-like targets. This numerical value is close to our result for $\tau$, which is based on charge- and spin-response functions for an interacting electron gas. Further, systematic calculations for real targets may provide additional information. This additional information, and further progress in the low-energy experimental techniques, would allow a meaningful comparison between the basic aspects of understanding.

(c) In order to show a detailed energy-dependence ( $E$ $\geqslant E_{F}$ ) of scattering rates $\tau_{c}$ and $\tau_{s}$, we have performed the double integration in Eqs. (1), (2) numerically at $r_{s}=5$ ( $K$ target) for an $E_{F} \leqslant E \leqslant 15 E_{F}$ energy range. The singleparticle excitations (particle-hole continuum) are treated precisely, and the plasmon (pl) contribution is calculated ${ }^{28}$ from the following expression:

$$
\frac{1}{\tau_{\mathrm{pl}}}=\frac{\omega_{p}}{\sqrt{2 E}} \ln \frac{\sqrt{E_{F}+\omega_{p}}-\sqrt{E_{F}}}{\sqrt{E}-\sqrt{E-\omega_{p}}},
$$

with $E \geqslant E_{F}+\omega_{p}$ where $\omega_{p}^{2}=4 \pi n_{0}$. Therefore, the minimum $E$ is prescribed here simply by the so-called single-pole ${ }^{2}$ value. Note, that the static local-field correction would lower the plasmon dispersion coefficient, shifting the RPA-based minimum energy closer to the single-pole estimation (see also Sec. III). The illustrative results are exhibited in Fig. 1, as a function of $x$. At higher energies the charge polarization

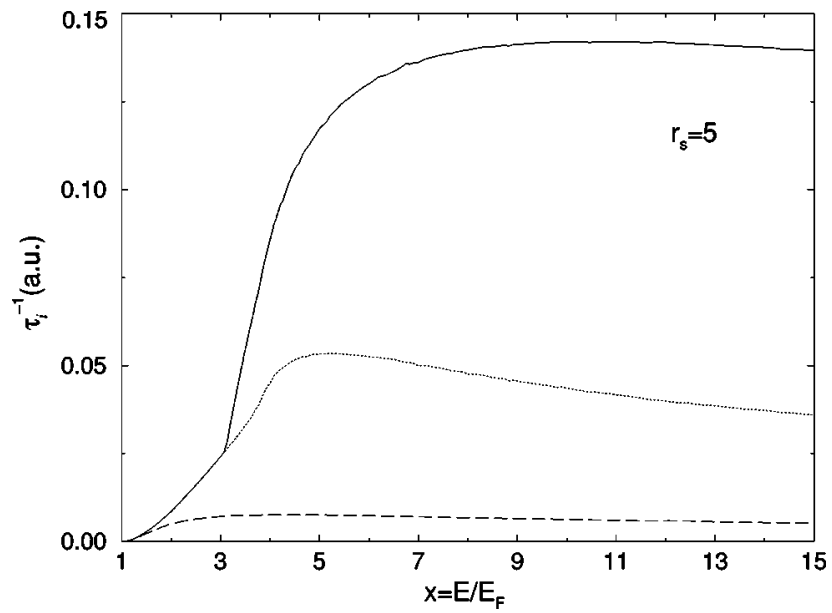

FIG. 1. The energy-dependence of $\tau_{i}^{-1}(i=c$, solid curve and $i=s$, dashed curve) obtained from Eqs. (1) and (2), respectively, at $r_{s}=5$. The dotted curve is the electron-hole-only contribution to $\tau_{c}^{-1}$. The rates are exhibited as a function of $x=E / E_{F}$.

channel (electron-hole plus plasmon) dominates. The plasmon channel results in a well-known, relatively sharp, upterm.

At lower energies, the important range for nontrivial correlation effects, the separated channels give comparable contributions to the net relaxation rate. This important lowenergy sector is shown in Fig. 2, for $r_{s}=4$ and $x \in[1,2]$. The solid and dashed curves are based on complete numerical integrations of Eqs. (1), (2), respectively, while the dotted and dash-dotted refer to Eq. (10) [with $a_{c}\left(r_{s}=4\right)=1.831$ ] and Eq. (9) [with $(2 C)^{2}=\pi a_{c}\left(r_{s}\right)$ ], respectively. One can see the usefulness of Eq. (9) in modeling the (screened) coupling to single-particle excitations, with a physically motivated estimation for $C$. Interestingly, Eq. (9) (with the $C$ value at $r_{s}=4$ ) could describe the magnitude and shape (for $x \in[1,9])$ of the fully self-consistent $G W$ result, plotted in Fig. 10 of Ref. 11. Further, transport, Knight-shift, and spec-

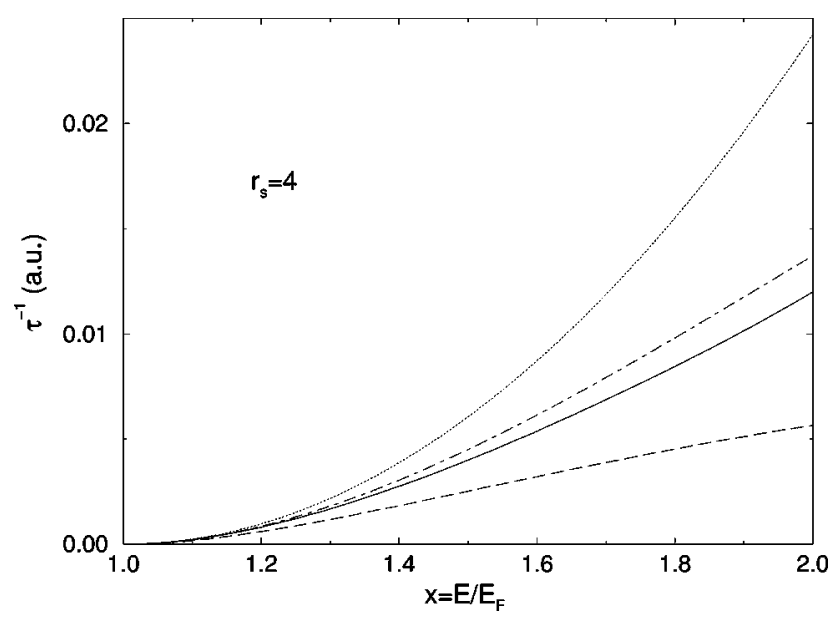

FIG. 2. The low-energy sector for the damping at $r_{s}=4$. Solid and dashed curves as in Fig. 1. The dotted curve refers to Eq. (10) with $a_{c}=1.831$. The dash-dotted refers to Eq. (9) with (see the text) the fixed $C$ value of $(2 C)^{2}=\pi a_{c}\left(r_{s}\right)$. 


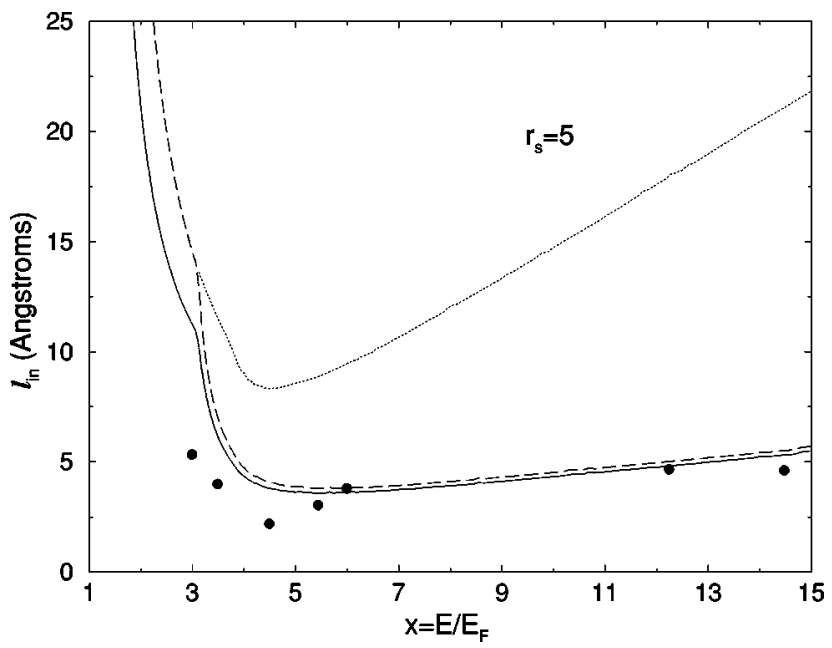

FIG. 3. Theoretical inelastic mean-free path $\left(l_{\text {in }}\right)$ curves obtained in different approximations, at $r_{s}=5$, as a function of $x$ $=E / E_{F}$. The solid curve refers to $\tau^{-1}=\tau_{c}^{-1}+\tau_{s}^{-1}$; the dashed one to the $\tau_{c}$-only channel; the dotted part to this channel without the collective contribution. The black dots are taken from an experimental paper (Ref. 30) on $K$ target. They were published without error bars. See text for further details.

troscopic experiments could help in our understanding on the physically most important (correlation-influenced) lowenergy sector.

A $\tau$-related quantity, the so-called inelastic mean-free path $l_{\text {in }}=\tau \sqrt{2 E},{ }^{29}$ is plotted in Fig. 3. The solid curve is our complete result obtained by using $\tau^{-1} \equiv \tau_{c}^{-1}+\tau_{s}^{-1}$. The dashed curve corresponds to the charge-only polarization case $\left(\tau_{c}\right)$ with electron-hole and plasmon excitations. The dotted part refers to this result but without the collective contribution. We note that the dashed curve is close to the $\tau_{\mathrm{RPA}}$-based version (not shown in the figure) for $l_{\text {in }}$; see Table I, also.

The black dots, for a $K$ target, are taken from an experimental paper where the data were plotted $^{30}$ by assuming an equivalence between the measured attenuation length $(\lambda)$ and the mean-free path $\left(l_{\text {in }}\right)$. Real physical effects (impurities, defects, phonons, pure dephasing) may have an influence on the value of the substracted length. This implies ${ }^{31}$ that the attenuation length is shorter than a theoretical meanfree path. In the light of this, one can consider our result as a meaningful ${ }^{29}$ one.

(d) In the rest of this section, we shall establish a link between the present [see point (b)] and the kinetic approaches for the lifetime. The low-energy limit of the RPA treatment (integrations over the wave vectors of energetically allowed excitations) gives a relaxation rate for the added electron (excited particle) that also emerges from the firstorder Born collisional ${ }^{19}$ treatment, based on particle-particle scattering without $^{4}$ exchange antisymmetrization, as was shown explicitly in a recent comparative study. ${ }^{32}$ The Born (B) collision method gives for the integrated relaxation rate

$$
\frac{1}{\tau_{B}}=\left(E-E_{F}\right)^{2} \frac{1}{(2 \pi)^{3}} \frac{1}{v_{F}} \int_{0}^{2 v_{F}} d q w(q),
$$

in which $w(q)$ is essentially a transition probability. It is expressed by a presupposed (spin-independent) $V_{\text {eff }}(q)$ interaction as $w(q)=\left|V_{\text {eff }}(q)\right|^{2}$. The above-mentioned important equivalence $\left(\tau_{B}=\tau_{\mathrm{RPA}}\right)$ refers to the natural choice of $V_{\mathrm{eff}}(q)=V_{\mathrm{RPA}}(q)=v(q) /\left[1+v(q) \chi_{0}(q)\right]$.

Precisely, it is this fact which suggests us to establish a similar link [via an $V_{\text {eff }}^{*}(q)$ ] with the unified low-energy result obtained above by using charge and spin polarization channels to $\tau^{-1}=\tau_{c}^{-1}+\tau_{s}^{-1}$. In order to achieve this goal we suppose a model (spin-conserving) particle-particle interaction of the usual ${ }^{33}$ form $V_{\mathrm{eff}}^{*}(r)=V_{1}(r)+\sigma_{1} \sigma_{2} V_{2}(r)$. Using a standard ${ }^{34}$ matrix-element calculation (a golden-rule treatment, mediated by two-electron spin functions in initial and final states) for the Born probability amplitudes and summation of the obtained partial (three) probabilities one can write

$$
\begin{aligned}
w^{*}(q)= & \frac{1}{2}\left[\left|V_{1}(q)+V_{2}(q)\right|^{2}\right. \\
& \left.+\left|V_{1}(q)-V_{2}(q)\right|^{2}+\left|2 V_{2}(q)\right|^{2}\right] .
\end{aligned}
$$

Here the last term is the spin-flip term and the (1/2) prefactor controls the proper weighting to an integrated rate. Simple comparison, based on Eq. (10) with Eqs. (1), (2) and Eqs. (11), (12), and a reinterpretation under the $\left(1 / \tau_{B}^{*}\right)=(1 / \tau)$ condition yields

$$
\begin{aligned}
& V_{1}(q)=\frac{V_{c}(q)}{1+V_{c}(q) \chi_{0}(q)}, \\
& V_{2}(q)=\frac{V_{s}(q)}{1+V_{s}(q) \chi_{0}(q)} .
\end{aligned}
$$

Importantly, the established nontrivial link between the different (polarization and scattering) treatments seems to be in complete agreement with the statement of MacDonald and Geldart on the form of an effective interaction to be used in the kinetic method without exchange antisymmetrization in scattering. ${ }^{35}$ Of course, the standard alternative method [with our $V_{\mathrm{eff}}^{*}(r)$ in channel-spin ${ }^{36}$ representation], based on singlet and triplet weighting, results in the same expressions because in this method

$$
w^{*}(q)=\frac{1}{4}\left|V_{1}(q)-3 V_{2}(q)\right|^{2}+\frac{3}{4}\left|V_{1}(q)+V_{2}(q)\right|^{2} .
$$

We stress the point that, in order to obtain Eqs. (14) and (15) the deductive way (by reinterpreting the low-energy limit of the results based on the adopted $G^{0} W$-type framework) was used. The residual particle-particle interaction (which describes the scattering lifetime at the Fermi surface) obtained in such a way becomes spin dependent. This is the expected behavior; ${ }^{2,19,33}$ it is due to statistical and dynamical correlations. The $V_{s}(q)=0$ dielectric, i.e., charge-onlyresponse treatment (see, above) would give $V_{2}(q)=0$.

The scattering interpretation, Eq. (13), shows that at lower densities the antiparallel-spin channels (second and third terms) dominate the integrated rate $1 / \tau_{B}^{*}$. An effective 
independent-particle picture emerges in which the role of indirect interactions (the charge and spin polarizations of the system itself) is treated on the average.

The equivalence allows an estimation for the spin-flip contribution $\left(\tau_{s f}^{-1}\right)$. Simple comparison gives $\left(1 / \tau_{s f}\right)$ $=(2 / 3)\left(1 / \tau_{s}\right)$ and thus, at lower densities, $1 / \tau_{s f}$ is in the order of $1 / \tau_{c}$. Furthermore, and this is one of our motivation, the short-range form (essentially a difference of Yukawa-like terms) of $V_{s}(r)$ from Eq. (4) allows an implementation ${ }^{37}$ in band-structure-based calculations for the electron-hole propagator $\left(\chi_{0}\right)$ of a paramagnetic system, via an appropriate treatment of Eq. (2).

\section{SUMMARY AND COMMENTS}

In the present paper we have investigated one of the theoretical methods for the relaxation rate in order to understand the influence of simultaneous charge and spin polarization effects on the phenomena. The physically appealing, excited particle plus medium with inherent degrees of freedom, framework allows a simple unified treatment for the paramagnetic electron gas.

One of our main results is the quantitative estimation for the relative contributions of different channels. For our model system the spin-channel turns out to be an important one, in the most important low-energy range. Similar quantitative estimations, using band-calculation-based response functions, might clarify a basic question in this field: the precise role and relative importance of band-related oneparticle and many-body effects. The applied, $G^{0} W$-type, framework suggests that this goal is accessible. Since paradigms (in the present case: a band or a Fermi-liquid picture) gain their status by rigid applications, comparative studies should have an important impact on our understanding.

Even at a linear-response level there are further subquestions for future investigations. (i) The influence of complex $G_{i}(q, \omega)$ local-field factors at low energies and their interplay (competition), through the $\operatorname{Im} \chi_{i}(q, \omega)$ functions, with the renormalization of the one-particle spectral weight codified by the one-particle Green's function. (ii) The effect of these $(i=c, s)$ dynamical factors in the plasmon damping ${ }^{14,38}$ in addition to the band-related ${ }^{10}$ damping mechanism.

From a more conceptual point of view the appealing picture of a dissipative medium, as an external complex field, is expected to be correct in first-order (linear) treatments. On the other hand, since in general the relaxation is related to the imaginary part of the proper four-point vertex function and this latter is governed ${ }^{5}$ by the corresponding BetheSalpeter equation, the single-screened interactions may be unreliable ones in a nonlinear coupling range. Therefore, a formal expansion in terms of linearly shielded interactions would result in an, presumably, asymptotic series only. ${ }^{11,24}$ The exchange-antisymmetrization in particle-particle scattering (interference) also requires refined considerations ${ }^{39}$ beyond a mean-field treatment.

\section{ACKNOWLEDGMENTS}

The work of one of us (I.N.) has been supported by the hungarian OTKA (Grant No. T034363). M.A. and P.M.E. acknowledge support from UPV/EHU and Eusko Jaurlaritza, Ministerio de Educación y Cultura (Grant No. PB97-0636), and Iberdrola S.A. Discussions with Professor E.V. Chulkov on a band-oriented extension are gratefully acknowledged.
${ }^{1}$ A. L. Fetter and J. D. Walecka, Quantum Theory of ManyParticle Systems (McGraw-Hill, New York, 1971).

${ }^{2}$ G. D. Mahan, Many-Particle Physics (Plenum, New York, 1981).

${ }^{3}$ J. C. Inkson, Many-Body Theory of Solids (Plenum, New York, 1984).

${ }^{4}$ L. P. Kadanoff and G. Baym, Quantum Statistical Mechanics (Benjamin, New York, 1962).

${ }^{5}$ J. M. Ziman, Elements of Advanced Quantum Theory (Cambridge University Press, Cambridge, 1969).

${ }^{6}$ I. Campillo, J.M. Pitarke, A. Rubio, E. Zarate, and P.M. Echenique, Phys. Rev. Lett. 83, 2230 (1999).

${ }^{7}$ W.D. Schöne, R. Keyling, M. Blandic, and W. Ekardt, Phys. Rev. B 60, 8616 (1999).

${ }^{8}$ P.M. Echenique, J.M. Pitarke, E.V. Chulkov, and A. Rubio, Chem. Phys. 251, 1 (2000).

${ }^{9}$ L. Hedin, J. Phys.: Condens. Matter 11, R489 (1999).

${ }^{10}$ W. Ku and A.G. Eguiluz, Phys. Rev. Lett. 82, 2350 (1999); B.C. Larson, J.Z. Tischler, E.D. Isaacs, P. Zschack, A. Fleszar, and A.G. Eguiluz, ibid. 77, 1346 (1996).

${ }^{11}$ B. Holm and U. von Barth, Phys. Rev. B 57, 2108 (1998).

${ }^{12}$ H. Yasuhara, S. Yoshinaga, and M. Higuchi, Phys. Rev. Lett. 83, 3250 (1999); M. Higuchi, S. Yoshinaga, and H. Yasuhara, J. Phys. Soc. Jpn. 68, 3473 (1999).
${ }^{13}$ W. Ku, A.G. Eguiluz, and E.W. Plummer, Phys. Rev. Lett. 85, 2410 (2000); H. Yasuhara, S. Yoshinaga, and M. Higuchi, ibid. 85, 2411 (2000).

${ }^{14}$ K. Sturm and A. Gusarov, Phys. Rev. B 62, 16474 (2000).

${ }^{15}$ H. Ehrenreich and M.H. Cohen, Phys. Rev. 115, 786 (1959).

${ }^{16}$ J. Lindhard, K. Dan. Vidensk. Selsk. Mat. Fys. Medd. 28 (8), 1 (1954).

${ }^{17}$ J.J. Quinn and R.A. Ferrell, Phys. Rev. 112, 812 (1958).

${ }^{18}$ R.H. Ritchie, Phys. Rev. 114, 644 (1959).

${ }^{19}$ D. Pines and P. Nozières, The Theory of Quantum Liquids (Benjamin, New York, 1966).

${ }^{20}$ C.A. Kukkonen and A.W. Overhauser, Phys. Rev. B 20, 550 (1979); X. Zhu and A.W. Overhauser, ibid. 33, 925 (1986).

${ }^{21}$ T.K. Ng and K.S. Singwi, Phys. Rev. B 34, 7738 (1986); 34, 7743 (1986).

${ }^{22}$ Y. Takada, Phys. Rev. B 47, 5202 (1993).

${ }^{23}$ K.S. Yi and J.J. Quinn, Phys. Rev. B 54, 13398 (1996).

${ }^{24}$ S. Yarlagadda and G.F. Giuliani, Phys. Rev. B 61, 12556 (2000).

${ }^{25}$ N. Iwamoto and D. Pines, Phys. Rev. B 29, 3924 (1984).

${ }^{26}$ A. Goldmann, W. Altmann, and V. Dose, Solid State Commun. 79, 511 (1991).

${ }^{27}$ I. Campillo, V.M. Silkin, J.M. Pitarke, E.V. Chulkov, A. Rubio, and P.M. Echenique, Phys. Rev. B 61, 13484 (2000). 
${ }^{28}$ J.J. Quinn, Phys. Rev. 126, 1453 (1962).

${ }^{29}$ L. Hedin and J.D. Lee, Phys. Rev. B 64, 115109 (2001).

${ }^{30}$ G.K. Wertheim, D.M. Riffe, N.V. Smith, and P.H. Citrin, Phys. Rev. B 46, 1955 (1992).

${ }^{31}$ J.J. Quinn, Appl. Phys. Lett. 2, 167 (1963).

${ }^{32}$ I. Nagy, J.I. Juaristi, and P.M. Echenique, Phys. Rev. B 63, 035102 (2000).

${ }^{33}$ A.J. Leggett, Rev. Mod. Phys. 47, 331 (1975).

${ }^{34}$ L. E. Ballentine, Quantum Mechanics (Prentice Hall, New York,
1990), pp. 323, 324.

${ }^{35}$ A.H. MacDonald and D.J.W. Geldart, Can. J. Phys. 60, 1016 (1982).

${ }^{36}$ G. R. Satcher, Direct Nuclear Reactions (Clarendon, Oxford, 1983), pp. 131-135.

${ }^{37}$ V. M. Silkin, E. V. Chulkov, and P. M. Echenique (unpublished).

${ }^{38}$ A.A. Kugler, J. Stat. Phys. 12, 35 (1975).

${ }^{39}$ I. Nagy, M. Alducin, J.I. Juaristi, and P.M. Echenique, Phys. Rev. B 64, 075101 (2001). 